\title{
Coherent and Incoherent Contributions to Multisoliton Complexes
}

\author{
Andrey A. Sukhorukov and Nail N. Akhmediev \\ Australian Photonics Cooperative Research Centre, Research School of Physical Sciences and Engineering, \\ Optical Sciences Centre, Australian National University, Canberra, Australian Capital Territory 0200, Australia
}

(Received 6 April 1999)

\begin{abstract}
We analyze multisoliton complexes and their dynamics in Kerr-like nonlinear media. The field in each of $M$ incoherently interacting components is calculated using an integrable set of coupled nonlinear Schrödinger equations. We obtain a general $N$-soliton solution describing propagation of multisoliton complexes and their collisions. The evolution of such higher-order soliton beams is determined by coherent and incoherent contributions from fundamental solitons. These internal interactions are revealed and illustrated by numerical examples. This theory can be applied to describe the properties of incoherent solitons in a photorefractive medium since these are, essentially, multisoliton complexes.

PACS numbers: 42.65.Tg, 05.45.Yv
\end{abstract}

One of the most noted discoveries of modern soliton science is that solitons can be excited by an incandescent light bulb instead of a high power laser source [1]. This produces "incoherent solitons" [1-3]; they can exist in photorefractive materials which require amazingly low powers to observe highly nonlinear phenomena [4-6]. It is also remarkable that, in certain conditions, incoherent solitons in photorefractive materials can be studied using coupled nonlinear Schrödinger equations (NLSE) [3,7].

In general, NLSE can be applied to various phenomena. These include incoherent solitons in photorefractive materials, plasma waves in random phase approximation [8], multicomponent Bose-Einstein condensate [9], and self-confinement of multimode optical pulses in a glass fiber [10]. Therefore its solutions are of great interest for theoretical physicists. In special cases these equations are found to be integrable [11]. Then, in analogy with single (scalar) NLSE (when the number of equations, $M$, is 1) [12] and the Manakov case [13] $(M=2)$, the total solution consists of a finite number $(N)$ of solitons and small amplitude radiation waves. The former is defined by the discrete spectrum of linear $(L, A)$ operators $[12,13]$ and the latter is defined by the continuous spectrum. Most applications deal with the soliton part of the solution as it contains the most important features of the problem. Moreover, a localized superposition of fundamental solitons can be called "multisoliton complex." An incoherent soliton is a particular example of a multisoliton complex [14].

The cases $M=1$ and $M=2$ have been extensively discussed in the literature $[12,13]$. On the other hand, results for general $M$ are scarce. The linear $(L, A)$ operators are important elements for the inverse scattering technique, which can be considered as a basis for integrability of $M$ coupled NLSEs. Moreover, it has been shown [15] that $N$ soliton solutions of $M$ coupled NLSE can be found using a simple technique which is an extension of the theory of reflectionless potentials [16]. In recent works [14,17] cases when each component has only one fundamental soliton have been considered. It was demonstrated that, in this configuration, the formation of stationary complexes may be observed, and corresponding solutions for $M=N \leq 4$ were presented in explicit form [17].

So far, only the case of complete mutual incoherence of the fundamental solitons has been considered. In this case the multisoliton complex can also be viewed as a selfinduced multimode waveguide [14]. The general case, where fundamental solitons in the multisoliton complex interact both coherently and incoherently, has not been analyzed. Such interactions may be observed if $N$ is larger than $M$, so that each component does not have less than one fundamental soliton. In general, each fundamental soliton can be "spread out" among several components. We will refer to this effect as mixed "polarization" of fundamental solitons. However, in order to capture distinctive features of coherent and incoherent soliton interactions, we will focus on a special case which is important for incoherent solitons. Specifically, we consider a situation where all the fundamental soliton polarizations are mutually parallel or orthogonal, and thus are conserved in collisions [14]. Because of the symmetry of the NLSE with respect to rotations in functional space, hereafter we assume that each fundamental soliton is polarized in one component only. It is for this case that we present new explicit $N$-soliton solutions of $M$ coupled NLSEs, and we discuss the new physics which it brings into the theory.

We consider propagation of an incoherent self-trapped beam in a slow Kerr-like medium and write the set of coupled NLSEs in the form $[3,7,14]$

$$
i \frac{\partial \psi_{m}}{\partial z}+\frac{1}{2} \frac{\partial^{2} \psi_{m}}{\partial x^{2}}+\delta n(I) \psi_{m}=0,
$$

where $\psi_{m}$ denotes the $m$ th component of the beam, $z$ is the coordinate along the direction of propagation, $x$ is the transverse coordinate, and

$$
\delta n(I)=\sum_{m=1}^{M} \alpha_{m}\left|\psi_{m}\right|^{2}
$$

is the change in refractive index profile created by all incoherent components of the light beam, where the $\alpha_{m}$ 
$(>0)$ are the coefficients representing the strength of the nonlinearity, and $M$ is the number of components.

Solutions in the form of multisoliton complexes of Eq. (1) and their collisions can be obtained using the formalism of $[15,18]$ with some refinements. First, we introduce functions $u_{j}(x, z)$ as solutions of the following set of equations:

$$
\sum_{m=1}^{N} D_{j m} u_{m}=-e_{j}
$$

where $N$ is a total number of fundamental solitons, $e_{j}=$ $\chi_{j} \exp \left(k_{j} \bar{x}_{j}+i k_{j}^{2} \bar{z}_{j} / 2\right), \bar{x}_{j}=x-x_{j}$, and $\bar{z}_{j}=z-z_{j}$ are shifted coordinates, and $\chi_{j}$ are arbitrary coefficients. The values $x_{j}$ and $z_{j}$ characterize the initial positions of fundamental solitons, but the actual beam trajectories may not follow the specified points due to mutual interactions between fundamental solitons. Each fundamental soliton is characterized by an eigenvalue $k_{j}=r_{j}+i \mu_{j}$. Its real part, $r_{j}$, determines the amplitude of the fundamental soliton, while the imaginary part, $\mu_{j}=\tan \theta_{j}$, accounts for the soliton velocity (i.e., motion in transverse direction). Here $\theta_{j}$ is the angle of the fundamental soliton propagation relative to the $z$ axis.

To distinguish coherent and incoherent contributions to the multisoliton complex, we use variables $n_{j}$, which represent the number of the component where the $j$ th soliton is located. Thus, two fundamental solitons with $n_{j}=n_{m}$ are coherent, and they are incoherent otherwise. Now we can write the expression for the matrix $D$ :

$$
D_{j m}=\frac{e_{j} e_{m}^{*}}{k_{j}+k_{m}^{*}}+ \begin{cases}1 /\left(k_{j}+k_{m}^{*}\right), & n_{j}=n_{m}, \\ 0, & n_{j} \neq n_{m} .\end{cases}
$$

Finally, the $N$-soliton solution of the original Eq. (1) can be obtained by adding up of all the $u_{j}$ corresponding to a given component number $m$ :

$$
\psi_{m}=\sum_{j ; n_{j}=m} u_{j} / \sqrt{\alpha_{m}} .
$$

Note that the number of terms in the sum is exactly the number of fundamental solitons polarized in this component, viz. $N_{m}$, and the total $N$ is $\sum_{m=1}^{M} N_{m}$.

One of the features of this approach is that coherent fundamental solitons are "split" among all the $u_{j}$ functions for a given component. However, when obtaining analytical solutions in explicit form, it is possible to separate fundamental solitons by combining terms with corresponding propagation constants. Consequently, we write the exact solutions for a different set of functions $\tilde{u}_{j}$, with each of them containing one fundamental soliton (at distances where coherent interactions are small). These are combined into the original functions in the following way: $\psi_{m}=\sum_{j ; n_{j}=m} \tilde{u}_{j} / \sqrt{\alpha_{m}}$.

The coefficients $\chi_{j}$ are arbitrary, and we can choose particular values for them:

$$
\chi_{j}=\prod_{m ; n_{m} \neq n_{j}} \sqrt{b_{j m}}
$$

where $b_{j m}=\left(k_{j}+k_{m}^{*}\right) /\left(k_{j}-k_{m}\right)$, and the square root value is taken on the branch with positive real part. This step significantly simplifies further analysis, as the resulting solution will acquire a highly symmetric form.

Finally, the explicit expressions for solutions can be found as sums over specific permutations:

$$
\begin{aligned}
\tilde{u}_{j} & =\frac{e^{i \gamma_{j}}}{U} \sum_{\{1, \ldots, j-1, j+1, \ldots, N\} \rightarrow L} C_{L}^{j} F_{L}^{j}(x, z), \\
U & =\sum_{\{1, \ldots, N\} \rightarrow L} C_{L} F_{L}(x, z) .
\end{aligned}
$$

Here $L$ denotes four sets of indices $\left(L_{1}, L_{2}, L_{3}, L_{4}\right)$. The summation is performed over all combinations in which the given set of soliton numbers (for example, $\{1, \ldots, N\}$ ) can be split among all the $L_{j}$. When performing permutations, $\left(L_{1}, L_{2}\right)$ are only filled with numbers of mutually coherent soliton pairs $\left(l_{1}, l_{2}\right)$ (thus the number of elements in these sets is the same).

The coefficients and functions from (7) are determined for each realization of the permutation $L$ as follows:

$$
\begin{aligned}
C_{L}= & (-1)^{\left|L_{1}\right|} T_{\mathrm{sg}}^{L_{1}} T_{\mathrm{sg}}^{L_{2}} T_{\mathrm{mg}} T_{\mathrm{sb}}^{L_{3}} T_{\mathrm{sb}}^{L_{4}} T_{\mathrm{mb}} T_{\mathrm{mgb}}, \\
F_{L}(x, z)= & \cos \left(S_{\mathrm{g}}\right) \cos \left(S_{\mathrm{f}}\right) \cosh \left(S_{\mathrm{b}}\right) \\
& -\sin \left(S_{\mathrm{g}}\right) \sin \left(S_{\mathrm{f}}\right) \sinh \left(S_{\mathrm{b}}\right), \\
C_{L}^{j}= & (-1)^{\left|L_{1}\right|} T_{\mathrm{c}}^{j} T_{\mathrm{sg}}^{L_{1}} T_{\mathrm{sg}}^{L_{2}} T_{\mathrm{mg}} T_{\mathrm{g}}^{j} T_{\mathrm{sb}}^{L_{3}} T_{\mathrm{sb}}^{L_{4}} T_{\mathrm{mb}} T_{\mathrm{mgb}}, \\
F_{L}^{j}(x, z)= & \cos \left(S_{\mathrm{g}}^{j}\right) \cos \left(S_{\mathrm{f}}\right) \cosh \left(S_{\mathrm{b}}^{j}\right) \\
& -\sin \left(S_{\mathrm{g}}^{j}\right) \sin \left(S_{\mathrm{f}}\right) \sinh \left(S_{\mathrm{b}}^{j}\right) .
\end{aligned}
$$

Here we used $\left|L_{l}\right|$ to denote the number of elements in the set. Note that the $F$ functions are written in the simplest form in terms of trigonometric and hyperbolic functions, due to the specific choice of coefficients in Eq. (6).

The variables introduced above are the following sums and products over the $L_{j}$ sets:

$$
\begin{aligned}
T_{\mathrm{c}}^{j} & =\left[1+\sum_{m \in L_{1} ; n_{m}=n_{j}} 1\right]^{-1}, \\
T_{\mathrm{sg}}^{L_{l}} & =\prod_{\{j, m\} \in L_{l} ; j<m} \begin{cases}\left|k_{j}-k_{m}\right|^{2}, & n_{j}=n_{m}, \\
s_{j m}\left|k_{j}+k_{m}^{*}\right|, & n_{j} \neq n_{m},\end{cases} \\
T_{\mathrm{mg}} & =\prod_{j \in L_{1} ; m \in L_{2}} \begin{cases}1 /\left|k_{j}+k_{m}^{*}\right|^{2}, & n_{j}=n_{m}, \\
s_{j m} /\left|k_{j}-k_{m}\right|, & n_{j} \neq n_{m},\end{cases} \\
T_{\mathrm{g}}^{j} & =\prod_{m \in L_{1} \cup L_{2} \cup L_{3} \cup L_{4}} \begin{cases}1 / c_{j m}, & n_{j}=n_{m}, \\
s_{j m} \sqrt{c_{j m}}, & n_{j} \neq n_{m},\end{cases} \\
T_{\mathrm{sb}}^{L_{l}} & =\prod_{\{j, m\} \in L_{l} ; j \leq m} \begin{cases}1 /\left(2 r_{j}\right), & j=m, \\
c_{j m}^{-2}, & n_{j}=n_{m}, \\
1, & n_{j} \neq n_{m},\end{cases}
\end{aligned}
$$




$$
\begin{aligned}
& T_{\mathrm{mb}}=\prod_{j \in L_{3} ;} \begin{cases}1, & n_{j}=L_{4} \\
c_{j m}, & n_{j} \neq n_{m}\end{cases} \\
& T_{\mathrm{mgb}}=\prod_{m_{1} \in L_{1} \cup L_{2}} \begin{cases}1 / c_{m_{1} m_{2}}, & n_{m_{1}}=n_{m_{2}}, \\
s_{m_{1} m_{2}} \sqrt{c_{m_{1} m_{2}}}, & n_{m_{1}} \neq n_{m_{2}},\end{cases} \\
& m_{2} \in L_{3} \cup L_{4} \\
& S_{\mathrm{g}}=\sum_{j \in L_{l}} \gamma_{j}-\sum_{j \in L_{2}} \gamma_{j}, \quad S_{\mathrm{b}}=\sum_{j \in L_{3}} \beta_{j}-\sum_{j \in L_{4}} \beta_{j}, \\
& S_{\mathrm{g}}^{j}=S_{\mathrm{g}}-i\left(S_{\mathrm{sg}}^{j, L_{1}}-S_{\mathrm{sg}}^{j, L_{2}}\right) \\
& S_{\mathrm{sg}}^{j, L_{l}}=\sum_{m \in L_{l}} \begin{cases}2 \eta_{j m}, & n_{j}=n_{m}, \\
\eta_{j m}, & n_{j} \neq n_{m},\end{cases} \\
& S_{\mathrm{b}}^{j}=S_{\mathrm{b}}+i\left(S_{\mathrm{sb}}^{j, L_{3}}-S_{\mathrm{sb}}^{j, L_{4}}\right), \\
& S_{\mathrm{sb}}^{j, L_{l}}=\sum_{m \in L_{l}} \begin{cases}2 \varphi_{j m}, & n_{j}=n_{m}, \\
\varphi_{j m} & n_{j} \neq n_{m},\end{cases} \\
& S_{\mathrm{f}}=S_{\varphi}^{L_{1}, L_{3}}+S_{\varphi}^{L_{2}, L_{4}}-S_{\varphi}^{L_{1}, L_{4}}-S_{\varphi}^{L_{2}, L_{3}}, \\
& S_{\varphi}^{L_{l 1}, L_{l 2}}=\sum_{j \in L_{l 1} ; m \in L_{l 2}} \begin{cases}2 \varphi_{j m}, & n_{j}=n_{m}, \\
\varphi_{j m}, & n_{j} \neq n_{m}\end{cases}
\end{aligned}
$$

Here the " $\cup$ " operator is used to merge the sets, and the variables $\beta_{j}+i \gamma_{j}=k_{j} \bar{x}_{j}+i k_{j}^{2} \bar{z}_{j} / 2$ (with $\beta_{j}$ and $\gamma_{j}$ real), $\eta_{j m}=\log \left[\left|\left(k_{j}-k_{m}\right)\left(k_{j}+k_{m}^{*}\right)\right|\right] / 2$, $c_{j m}=\left|b_{j m}\right|, \quad \varphi_{j m}=\arg \left(1 / b_{j m}\right) / 2, \quad s_{j m}=\operatorname{sgn}\{\pi-$ $\left.\arg \left[\sqrt{b_{j m}}\left(\sqrt{b_{m j}}\right)^{*} /\left(k_{j}+k_{m}^{*}\right)\right]\right\} \operatorname{sgn}(m-j)$. The function arg is supposed to give values in the interval $[0,2 \pi)$, and

$$
\operatorname{sgn}(\xi)= \begin{cases}1, & \xi \geq 0 \\ -1, & \xi<0\end{cases}
$$

Note that only $\beta_{j}$ and $\gamma_{j}$ depend on the coordinates $(x, z)$. All the other coefficients are expressed in terms of the wave numbers $k_{j}$ and constant shifts in positions $\left(x_{j}, z_{j}\right)$ of the $N$ fundamental solitons. As the total solution has translational symmetry, one of the shifts can be fixed, so that the number of independent parameters controlling the multisoliton complex is $2 N-1$.

If an incoherent soliton consists only of orthogonally polarized fundamental solitons $\left(n_{j} \equiv j, N \equiv M\right)$, and all are propagating in the same direction, then its transverse intensity profile remains stationary [17]. In this particular case, the general expressions (8) are radically simplified, since, due to the above-mentioned restrictions on the permutations, the sets $L_{1}$ and $L_{2}$ are always empty. Hence, we obtain

$$
\begin{aligned}
& C_{L}=T_{\mathrm{mb}}, \quad C_{L}^{j}=2 r_{j} \chi_{j} T_{\mathrm{mb}}, \\
& F_{L}=\cosh \left(S_{\mathrm{b}}\right), \quad F_{L}^{j}=\cosh \left(S_{\mathrm{b}}^{j}\right) .
\end{aligned}
$$

Note that here we have neglected a common multiplier in $C_{L}$ and $C_{L}^{j}$, as these coefficients determine, respectively, the denominator and numerator in the expression for $\tilde{u}_{j}$.

Now we present numerical examples to illustrate these results. An example of a stationary incoherent soliton consisting of eight components $(N=M=8)$ is shown in Fig. 1. The profiles of the constituent fundamental solitons, and their superposition as a whole, are determined by the wave numbers and relative shifts along the $x$ axis. In this configuration, the shifts in propagation direction, $z_{j}$, correspond to arbitrary phase changes of different components, but these do not influence the evolution due to the incoherent nature of the intercomponent interactions.

On the other hand, if $N>M$, two or more of the fundamental solitons are polarized in the same components, and thus interact coherently. If the inclination angles of the fundamental solitons are all the same, the beam will remain localized upon propagation. Such a multisoliton complex is an incoherent soliton with an intensity profile which evolves periodically or quasiperiodically, as shown in Fig. 2. These oscillations, appearing due to internal coherent intracomponent interactions, are a general feature of incoherent solitons, and can be eliminated only in specific cases, as discussed earlier. It follows that spatial "beating" always accompanies the interaction of fundamental solitons of a single NLSE, which agrees with previous studies [19].

Our explicit solution (7) also describes collisions of incoherent solitons. As mentioned earlier, the polarizations of the fundamental solitons are preserved in collisions (provided they are orthogonal or parallel), and thus the degree of internal coherence does not change. However, the shifts of the fundamental soliton trajectories differ, and this results in the incoherent solitons changing

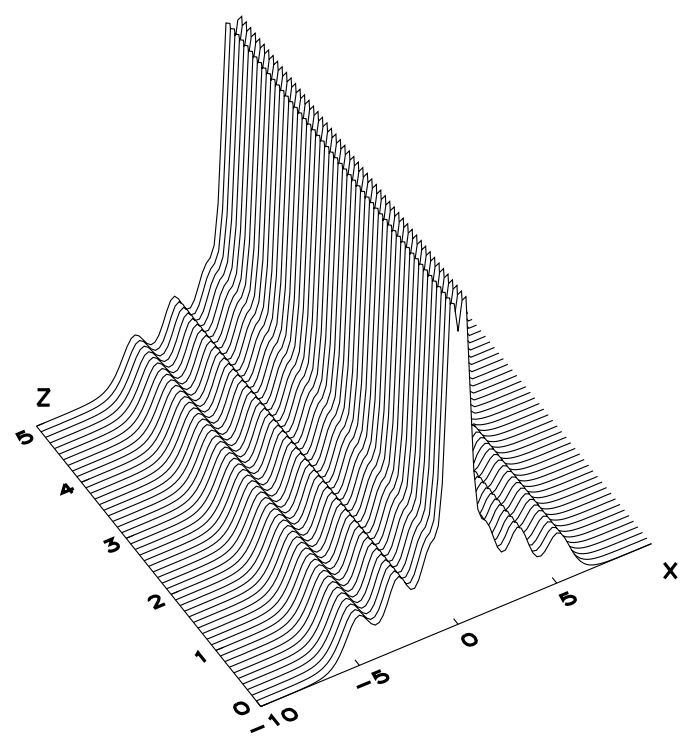

FIG. 1. Stationary propagation of an incoherent soliton consisting of eight completely incoherent fundamental solitons (polarized in different components). 


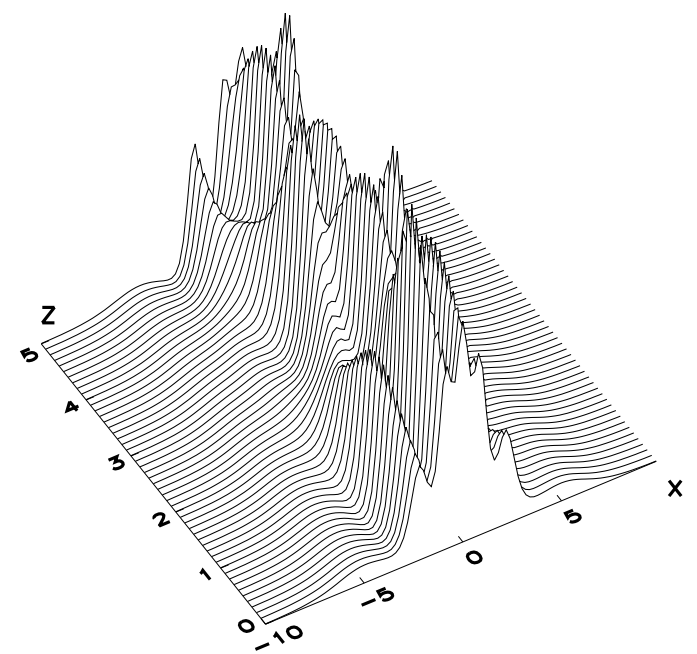

FIG. 2. Evolution of an incoherent soliton with multiscale periodic "beating" due to internal coherent interactions (eight fundamental solitons in five components).

their shapes. These transformations can be seen clearly in Fig. 3.

To calculate the shifts, we use the fact that in the expression for soliton profiles, $\tilde{u}_{j}$, given by Eq. (7), the denominator $U$ is real, and the numerator does not depend on the coordinates of the corresponding fundamental soliton $\left(x_{j}, z_{j}\right)$. It is then straightforward to take appropriate limits and calculate the shift of $j$ th fundamental soliton along the $x$ axis due to collisions:

$$
\delta x_{j}=\frac{1}{r_{j}} \sum_{m} \pm \begin{cases}2 \log \left(c_{j m}\right), & n_{j}=n_{m} \\ \log \left(c_{j m}\right), & n_{j} \neq n_{m}\end{cases}
$$

Here the summation involves the fundamental solitons which feature in the collisions. The "+" sign corresponds to the case when colliding soliton number $m$ comes from the right (i.e., has a larger $x$ coordinate before the

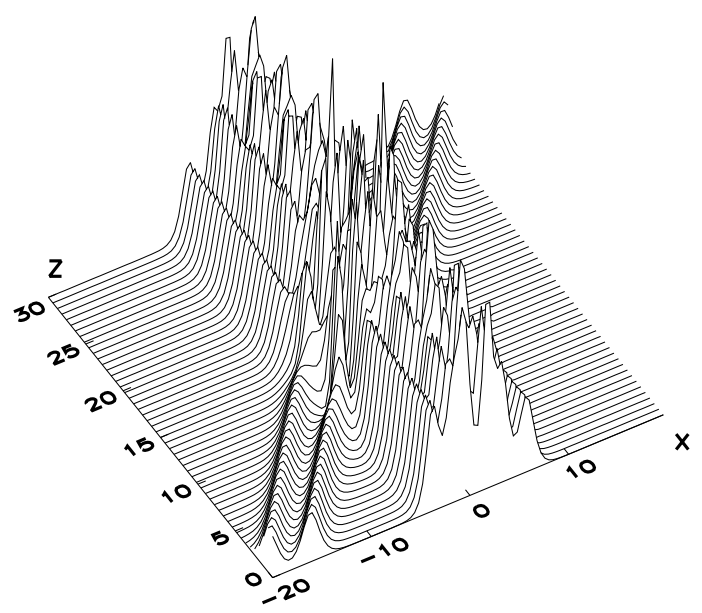

FIG. 3. Collision of a completely incoherent soliton (consisting of two orthogonally polarized fundamental solitons) and an incoherent soliton with internal coherent contributions (six fundamental solitons in five components). impact), and the "-" sign when from the left. This is a generalization of the expressions found in [17].

In summary, we have obtained a general $N$-soliton solution of $M$ coupled nonlinear Schrödinger equations which describes multisoliton complexes supported by a Kerr-type nonlinearity. A particular example is an incoherent soliton in a photorefractive medium. We have revealed that the properties of multisoliton complexes, which are superpositions of fundamental solitons with orthogonal or parallel polarizations, are determined by internal interactions, both phase-insensitive intercomponent and coherent intracomponent, with the latter resulting in spatial "beating." Using our exact result, we also analyzed collisions of incoherent solitons. We showed that the reshaping of incoherent solitons after collisions is characterized by the relative shifts of the fundamental solitons, and these are calculated using a simple analytical formula. These distinctive features of incoherent solitons are illustrated by numerical examples.

The authors are part of the Australian Photonics CRC. We are grateful to Dr. Ankiewicz for a critical reading of this manuscript.

[1] M. Mitchell and M. Segev, Nature (London) 387, 880 (1997).

[2] M. Mitchell, Z. Chen, M. Shih, and M. Segev, Phys. Rev. Lett. 77, 490 (1996).

[3] M.I. Carvalho, T.H. Coskun, D.N. Christodoulides, M. Mitchell, and M. Segev, Phys. Rev. E 59, 1193 (1999).

[4] G. Duree et al., Phys. Rev. Lett. 71, 533 (1993).

[5] A. A. Zozulya, D.Z. Anderson, A.V. Mamaev, and M. Saffman, Europhys. Lett. 36, 419 (1996).

[6] M.D. Iturbe-Castillo et al., Appl. Phys. Lett. 64, 408 (1994).

[7] V. Kutuzov, V.M. Petnikova, V.V. Shuvalov, and V. A. Vysloukh, Phys. Rev. E 57, 6056 (1998).

[8] A. Hasegawa, Phys. Fluids 20, 2155 (1977).

[9] E. P. Bashkin and A.V. Vagov, Phys. Rev. B 56, 6207 (1997).

[10] A. Hasegawa, Opt. Lett. 5, 416 (1980).

[11] V. G. Makhan'kov and O. K. Pashaev, Teor. Mat. Fiz. 53, 55 (1982) [Theor. Math. Phys. 53, 979 (1982)].

[12] V.E. Zakharov and A. B. Shabat, Zh. Eksp. Teor. Fiz. 61, 118 (1971) [Sov. Phys. JETP 34, 62 (1971)].

[13] C. B. Manakov, Zh. Teor. Eksp. Fiz. 65, 505 (1973) [Sov. Phys. JETP 38, 248 (1974)].

[14] N. Akhmediev, W. Królikowski, and A. W. Snyder, Phys. Rev. Lett. 81, 4632 (1998).

[15] Y. Nogami and C. S. Warke, Phys. Lett. 59A, 251 (1976).

[16] I. Kay and H.E. Moses, J. Appl. Phys. 27, 1503 (1956).

[17] A. Ankiewicz, W. Królikowski, and N. N. Akhmediev, Phys. Rev. E 59, 6079 (1999).

[18] C. S. Gardner, J.M. Greene, M.D. Kruskal, and R.M. Miura, Commun. Pure Appl. Math. 27, 97 (1974).

[19] N. Akhmediev and A. Ankiewicz, Solitons, Nonlinear Pulses and Beams (Chapman Hall, London, 1997). 